\title{
Alzheimer disease therapy: Can the amyloid cascade be halted?
}

\author{
Todd E. Golde \\ Department of Neuroscience, Mayo Clinic Jacksonville, Jacksonville, Florida, USA \\ J. Clin. Invest. 111:11-18 (2003). doi:10.1172/JCI200317527.
}

In the not too distant future, clinical management of Alzheimer disease (AD) is likely to resemble the present management of atherosclerotic disease. Sometime before an individual reaches age 50, an internist will initiate a screening program to determine that person's risk for developing AD. This assessment will include a comprehensive genetic screen for $\mathrm{AD}$-risk loci, determination of plasma amyloid $\beta$ peptide $(A \beta)$ levels, family history of $\mathrm{AD}$, and, perhaps, potential environmental risks. Depending on the risk prediction, a follow-up visit with an Alzheimer specialist may be scheduled. During this visit, an amyloid-binding agent will be injected and used to evaluate the extent of amyloid deposition in the brain. Based on the amount of deposition present and the initial risk assessment, the specialist will then develop a personalized therapeutic regimen. This regimen might consist of an $A \beta$ vaccination, an amyloid-lowering drug, an anti-inflammatory agent, a neuronal growth factor, an antioxidant, or a combination of these approaches. The efficacy of therapy will be monitored by measurement of plasma $A \beta$ levels, imaging of amyloid in the brain, and volumetric scanning of the brain. Primary screening, along with monitoring of the presymptomatic indicators of disease, and appropriate intervention will significantly reduce one's risk for developing AD.

Although proposal of such a scenario might seem highly speculative, recent and expected advances in (a) understanding the pathogenesis of $\mathrm{AD}$, (b) identifying the genetic factors that confer risk for $\mathrm{AD}$, (c) validating potential biomarkers for $\mathrm{AD}$, and (d) developing therapeutic agents that target both $A \beta$ and downstream pathological changes greatly increase the likelihood that $\mathrm{AD}$ will be managed successfully in the future.

\footnotetext{
Address correspondence to: Todd E. Golde, Department of Neuroscience, Mayo Clinic Jacksonville, 4500 San Pablo Road, Jacksonville, Florida 32224, USA. Phone: (904) 953-2538; Fax: (904) 953-7370; E-mail: tgolde@mayo.edu.

Conflict of interest: The author has declared that no conflict of interest exists.

Nonstandard abbreviations used: Alzheimer disease (AD); amyloid $\beta$ peptide $(\mathrm{A} \beta)$; late-onset $\mathrm{AD}(\mathrm{LOAD})$; amyloid $\beta$ protein precursor (APP); secreted derivative of amyloid $\beta$ protein precursor (sAPP); carboxy-terminal fragment (CTF); presenilin (PS); British familial dementia (BFD); familial Danish dementia (FDD); neurofibrillary tangle (NFT); frontotemporal dementia with parkinsonism (FTDP); $\beta$-secretase (BACE1, for $\beta$-site APP-cleaving enzyme); Food and Drug Administration (FDA); cerebrospinal fluid (CSF).
}

\section{$A D$ is the leading cause of dementia in the elderly}

Estimates of prevalence vary, but $1-5 \%$ of the population over age 65 , and $20-40 \%$ of the population over age 85 , may be affected by $\mathrm{AD}$ (1). Given the increasing number of elderly individuals in industrialized societies, $\mathrm{AD}$ represents a burgeoning epidemic that exacts a tremendous toll on the individuals it affects, along with their families and caregivers. Moreover, $\mathrm{AD}$ has a tremendous negative economic impact amounting to over $\$ 100$ billion a year. Treatment of AD in the US reportedly costs more per patient than management of other major age-associated diseases $(2,3)$.

Beginning with short-term memory loss, and continuing with more widespread cognitive and emotional dysfunction, typical late-onset $\mathrm{AD}$ (LOAD) occurs after age 65 and follows an insidious 5- to 15-year course. Although AD usually presents without motor or sensory alterations, rare variants (such as spastic paraparesis) with atypical clinical presentations are occasionally recognized $(4,5)$. Even today, definitive diagnosis of $\mathrm{AD}$ is only possible through postmortem analysis of the brain (1). This histopathological analysis of the brain demonstrates the classic triad of $\mathrm{AD}$ pathology: (a) senile plaques containing $A \beta$, (b) neurofibrillary tangles (NFTs) containing tau, and (c) widespread neuronal loss in the hippocampus and select cortical and subcortical areas.

\section{Toward a complete understanding of $A D$ pathogenesis: is $A \beta$ the cholesterol of $A D$ ?} $A \beta$ accumulation as the initiating factor in $A D$ pathogenesis. Much of the $A \beta$ that accumulates in the $\mathrm{AD}$ brain is deposited as amyloid within senile plaques and cerebral vessels. Although numerous proteins are associated with the amyloid deposits in $\mathrm{AD}$, the principal proteinaceous component of $\mathrm{AD}$ amyloid is the approximately $4-\mathrm{kDa} A \beta . A \beta$ is produced from the amyloid $\beta$ protein precursor (APP) through two sequential proteolytic cleavages made by enzymes referred to as secretases (Figure 1) (6). APP is first cleaved at the amino-terminus of $A \beta$ by a membranebound aspartyl protease (referred to as $\beta$-secretase). This cleavage generates a large secreted derivative (sAPP $\beta$ ) and a membrane-bound APP carboxy-terminal fragment (CTF $\beta$ ). Cleavage of CTF $\beta$ by $\gamma$-secretase results in the production of $A \beta$ peptides of varying length. The two species of most interest are a 
40 -amino acid $A \beta$ peptide $(A \beta 40)$ and a 42 -amino acid $A \beta$ peptide $(A \beta 42)$. At the same time, a cognate CTF $\gamma$ is produced. Two homologous polytopic membrane proteases, referred to as presenilins 1 and 2 (PS1 and PS2), are likely $\gamma$-secretases. If they are not $\gamma$-secretases, PSs are at least essential cofactors for this cleavage (7).

A great deal of evidence that $A \beta$ is not a disease marker, but that it plays a causal role in the development of $\mathrm{AD}$ pathology, emerges from a variety of genetic, pathological, and biochemical studies (8-10). These studies demonstrate, first, that mutations in the APP, PS1, and PS2 genes that are linked to the earlyonset forms of familial $A D$ increase total $A \beta$, specifically increase the relative amount of long $A \beta$ peptides ending at $A \beta 42$, or alter the primary sequence of $A \beta$ so that it is more fibrillogenic $(11,12)$. Second, apoE4, a major risk factor in typical LOAD, increases $A \beta$ deposition (13). Third, $A \beta 42$ deposits as amyloid more rapidly than do shorter species (14). Fourth, overexpression of familial AD-linked mutant APPs, or coexpression of mutant APP and familial AD-linked PSs in the brains of transgenic mice, leads to $A \beta$ deposition and other $A D$-like pathology (15). Fifth, $A \beta$ can self-aggregate in vitro, and these aggregates can directly and indirectly mediate neurotoxicity (16).

Recent evidence from the study of British familial dementia (BFD) and familial Danish dementia (FDD) provides further support for the hypothesis that $A \beta$ accumulation in the brain is the cause of AD. BFD and FDD are late-onset dementing disorders characterized by non- $\mathrm{A} \beta$ plaques, tangles, and neuronal loss. $\mathrm{As}$ in $\mathrm{AD}$, the primary defect in BFD appears to be the abnormal generation of a peptide with amyloidogenic properties $(17,18)$. Thus, in two additional disorders, accumulation of an amyloidogenic peptide is linked to dementia.

Collectively, these data support a modified version of the amyloid cascade hypothesis (19). This modified cascade is schematically illustrated in Figure 2. Initially, investigators proposed that $A \beta$ accumulation as amyloid triggered a pathological cascade that ultimately produced the complete pathological and clinical symptoms of $\mathrm{AD}$. Today, this hypothesis remains valid, except that it is less clear whether $A \beta$ deposited as amyloid, or some other less well characterized $A \beta$ aggregate, initiates the cascade leading to neuronal death and dysfunction. Indeed, small $A \beta$ oligomers (20), also referred to as $A \beta$-derived diffusible ligands (ADDLs), and protofibrils $(21,22)$ have emerged as alternative aggregated forms of $A \beta$ that may mediate toxicity. Moreover, there is some evidence that intracellular accumulations of $A \beta$ may also be neurotoxic $(23,24)$. In any case, the evidence that $A \beta$ accumulation initiates $\mathrm{AD}$ pathology provides a framework in which to develop rational approaches for $\mathrm{AD}$ therapy based on altering $A \beta$ accumulation (Figure 1).

Downstream effects of $A \beta$ accumulation. At the present time, the $A \beta$-induced pathological cascades leading to neuronal dystrophy and death are not nearly as well defined as the pathways leading to $A \beta$ generation and accumulation. This might be attributable to the failure of animal models to fully recapitulate the pathological

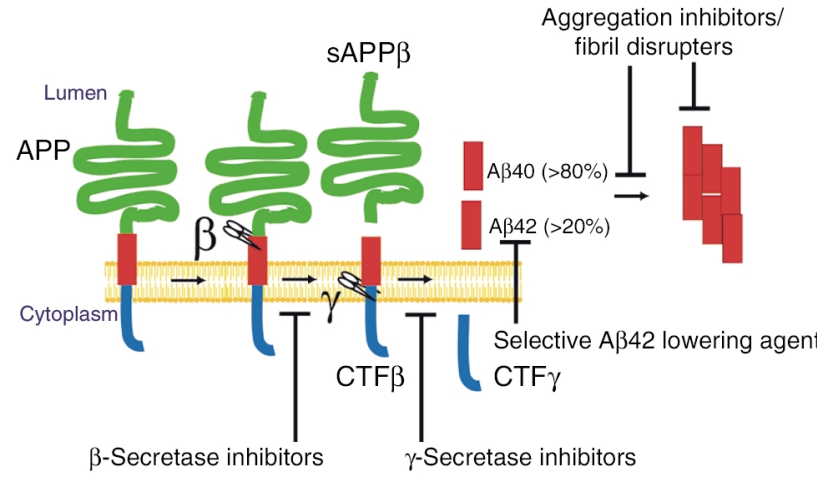

Figure 1

$A \beta$ generation, aggregation, and sites for therapeutic intervention. APP is a type I transmembrane protein that is processed in several different pathways. The $A \beta$ generation pathway is shown. Generation of $A \beta$ in the $\beta$-secretase pathway requires two proteolytic events, a proteolytic cleavage at the amino-terminus of the $A \beta$ sequence, referred to as $\beta$-secretase cleavage, and a cleavage at the carboxy-terminus, known as $\gamma$-secretase cleavage, which results in another carboxy-terminal fragment (CTF $\gamma$ ). Although many $A \beta$ peptides of various lengths can be produced in this fashion, the two of most interest are $A \beta 40$, which is the predominant $A \beta$ peptide, and $A \beta 42$, which is typically produced at much lower levels than $A \beta 40$. Although both peptides can aggregate, $A \beta 42$ is thought to aggregate much more rapidly and to seed the aggregation of $A \beta 40$. Sites for anti-A $\beta$ intervention are indicated. Scissors indicate proteolytic cleavages. "sAPP $\beta$ " refers to the large secreted derivative generated by $\beta$-secretase cleavage of APP.

features of $\mathrm{AD}$, especially with respect to the development of NFTs and neuronal cell death (25). Multiple pathways are also likely to mediate $A \beta$ toxicity. There is evidence that $A \beta$ can be directly neurotoxic, induce oxidative stress, incite an inflammatory response, and alter calcium homeostasis. These events might be mediated by direct interaction of $A \beta$ aggregates with cellular membranes, or by the binding of $A \beta$ to microglial and neuronal cellular receptors (26-28).

One important downstream event in the $A \beta$-induced cascade appears to be the development of neurofibrillary pathology. NFTs are intracellular inclusions composed of approximately $10-\mathrm{nm}$ paired helical filaments (PHFs). PHFs themselves are composed of hyperphosphorylated bundles of the microtubule-binding protein tau (29). Abnormal accumulations of hyperphosphorylated tau are also seen in the swollen, tortuous, neuritic processes often, but not exclusively, found in association with senile plaques. Genetic evidence from the study of frontotemporal dementia with parkinsonism linked to chromosome 17 (FTDP-17) indicates that mutations in tau cause this disease, which is characterized by cell death and neurofibrillary pathology (30, 31). Moreover, overexpression of FTDP mutant tau is sufficient to induce NFT pathology and neurofibrillary degeneration in transgenic mice $(32,33)$. In such mice, $A \beta$ injection or crossing of the tau mutant mice with APP transgenic mice results in enhanced NFT formation $(33,34)$. Significantly, FTDP-linked tau mutations do not cause alterations in $A \beta$. Thus, it appears that tau pathology is an important aspect of the pathological cascade induced by $A \beta$. Therapies aimed at modulating 
the $A \beta$-induced changes that lead to NFT formation are likely to be of some benefit in AD. Unfortunately, these pathways are not well defined, and no inhibitors of this process have been identified.

The rationale for anti-A $\beta$ therapy. Based on this evidence, it is not unreasonable to propose that the role $A \beta$ plays in $\mathrm{AD}$ is akin to the role cholesterol plays in atherosclerotic disease. Age-associated accumulation of either triggers a complex pathological lesion that, after a long period of time, results in clinical symptoms. Moreover, just as numerous factors contribute to cholesterol deposition, numerous factors can also influence $A \beta$ accumulation. Finally, just as lowering cholesterol levels has proven beneficial in the management of atherosclerotic disease, therapies aimed at reducing $A \beta$ accumulation are likely to be effective in preventing $\mathrm{AD}$. It is likely that the reduction in total $A \beta$ levels need not be complete in order to have some benefit if preventive therapy can be initiated. In AD caused by most mutations in APP, PS1, or PS2, A 442 levels are increased by as little as $30 \%$ (12). Such an elevation can result in the onset of AD 30-40 years earlier than typical LOAD. By inference, it is likely that reducing total $\mathrm{A} \beta$ levels by $30 \%$, or effecting similar selective reductions in the highly pathogenic $A \beta 42$, may delay the development of AD to such an extent that it is no longer a major health care problem.

\section{Anti-A $\beta$ therapies under development}

Secretase inbibitors. Research clarifying the metabolic pathways that regulate $A \beta$ production has revealed that the secretases that produce the $A \beta$ may be good therapeutic targets since inhibition of either $\beta$ - or $\gamma$-secretase decreases $A \beta$ production. More progress has been made in developing $\gamma$-secretase inhibitors, because highthroughput screens carried out in the pharmaceutical industry have identified numerous $\gamma$-secretase inhibitors. Multiple classes of potent $\gamma$-secretase inhibitors have now been described, and several of these have been shown to target both PS1 and PS2 (refs. 35-38; reviewed in ref. 7). At least one $\gamma$-secretase inhibitor is in clinical trials. Moreover, treatment of mice with a $\gamma$-secretase inhibitor reduces $A \beta$ levels in the brain and attenuates $A \beta$ deposition (39). However, despite these advances, numerous concerns over the use of $\gamma$-secretase inhibitors as $\mathrm{AD}$ therapeutics remain. These concerns center on target-mediated toxicity caused by interference with $\gamma$-secretase-mediated Notch signaling $(40,41)$; inhibition of signaling mediated by newly recognized $\gamma$-secretase substrates (such as the epidermal growth factor receptor ErbB4) or unrecognized substrates (42); or accumulation of potentially neurotoxic APP CTF $\beta$, which invariably occurs when $\gamma$-secretase is inhibited $(43,44)$.

Although the development of $\beta$-secretase inhibitors has lagged behind the development of $\gamma$-secretase inhibitors, many believe that $\beta$-secretase is likely to be a better therapeutic target. $\beta$-Secretase (BACE1, for $\beta$-site APP-cleaving enzyme) knockout mice produce no A $\beta$, yet they have no obvious pathological phenotype (45, 46). Significantly, the crystal structure of BACE1 has been solved $(47,48)$. Such structural information will surely speed the drug discovery efforts, currently underway, to develop potent nonpeptidic BACE1 inhibitors. Although the knockout studies partially allayed fears that BACE1 inhibition might be problematic due to inhibition of cleavage of non-APP substrates, concerns remain regarding target-mediated toxicity. Moreover, the crystal structure of BACE1 reveals a wide, active-site gorge that may be difficult to target with small-molecule inhibitors $(47,48)$.

Very recently, several Food and Drug Administration-approved (FDA-approved) NSAIDs, including ibuprofen, sulindac, and indomethacin, have been shown to be selective A $\beta 42$-lowering agents (49). More-

\section{Figure 2}

$A \beta$ aggregation as the cause of $A D$. A modified version of the amyloid cascade hypothesis is shown. This version takes into account the possibility that $A \beta$ aggregates other than those found in classic amyloid deposits initiate the pathological cascade. It is possible that $A \beta$-induced toxicity in turn results in alterations in the brain, such as increased APP and apoE expression, that enhance $A \beta$ deposition, although this is not shown in the figure. Besides known genetic pathways, a pathway in which normal $A \beta$ levels in the context of normal aging may lead to $A \beta$ accumulation is shown. "APPSw" refers to the APP Swedish mutant linked to familial AD; this mutation alters the lysine-methionine sequence immediately preceding $A \beta$ to asparagine-leucine. Trisomy 21 is also known as Down syndrome.

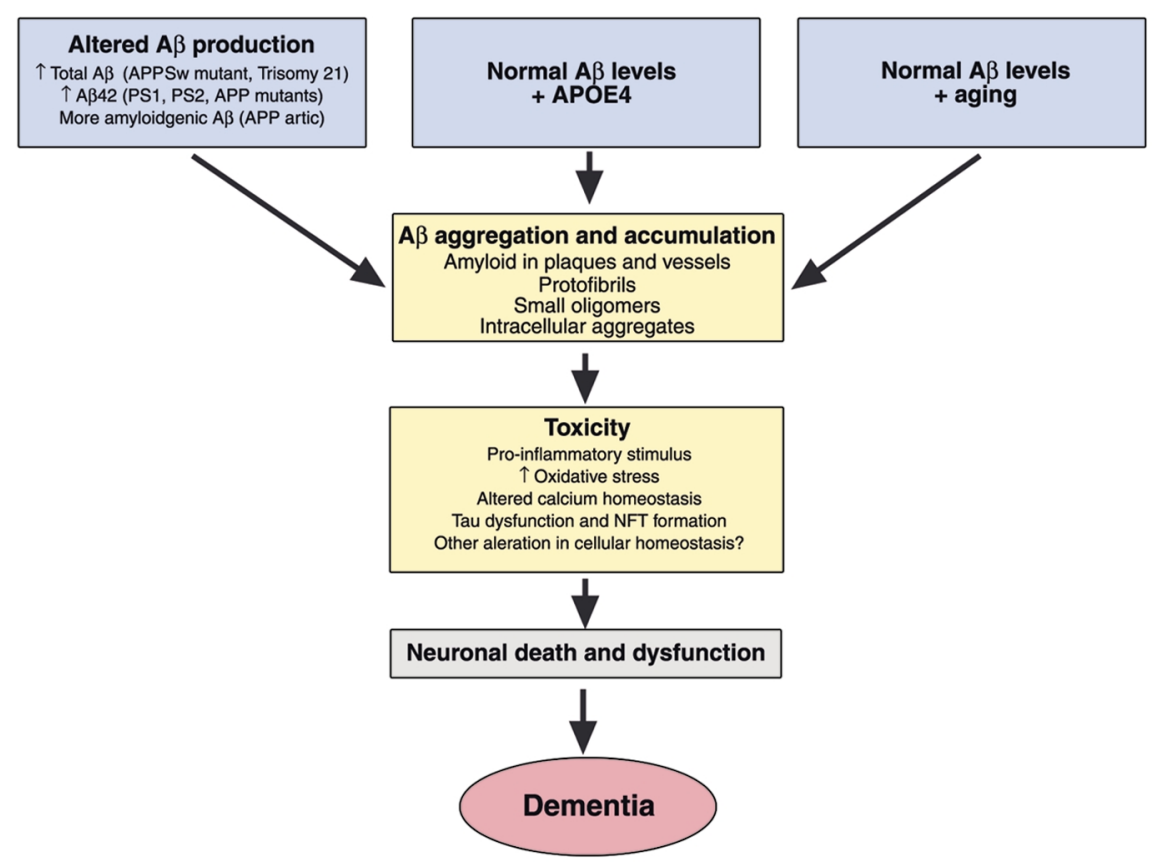


over, long-term treatment of APP transgenic mice with ibuprofen attenuates $A \beta$ deposition (50). Although the mechanisms by which these NSAIDs lower $A \beta 42$ have not been established, the effect is independent of cyclooxygenase inhibition, which is the primary antiinflammatory target of these compounds (49). These substances do not change the total level of $A \beta$ produced but, rather, shift cleavage from $A \beta 42$ to a shorter 38 -amino acid $A \beta$ peptide $(A \beta 38)$. This finding suggests that they are interacting with $\gamma$-secretase. Although the contribution of $\mathrm{A} \beta 38$ to AD pathology is not known, it is generally accepted that $A \beta 42$ is the pathogenic $A \beta$ species (9). Therefore, investigators believe that lowering $A \beta 42$ levels is a therapeutic strategy worthy of further investigation. The implications of these findings, with respect to the therapeutic potential of anti-inflammatory agents, will be discussed shortly.

Cholesterol-altering drugs. Epidemiologic data and data from model systems indicate that cholesterol-altering drugs may have an impact on the development of AD, and that this effect could be attributed to effects on $A \beta$ accumulation. Retrospective studies on $\beta$-hydroxy- $\beta$ methylglutaryl-coenzyme A (HMG-CoA) reductase inhibitors (statins) show a large reduction in the risk for developing AD in individuals taking these drugs $(51,52)$, whereas individuals with elevated cholesterol are at higher risk for the development of AD (53-56). In culture and animal model systems, statins and other cholesterol-lowering agents decrease $A \beta$ levels and $A \beta$ deposition (57-59), whereas high-cholesterol diets in APP transgenic mice increase A $\beta$ deposition (60). In addition, inhibitors of acetyl coenzyme A:cholesterol acyltransferase (ACAT), the enzyme that converts free cholesterol to cholesterol esters, also appear to decrease $\mathrm{A} \beta$ production (61).

Cholesterol's role in $\mathrm{A} \beta$ metabolism appears to be quite complex and is the subject of recent reviews (62, 63). Cholesterol-modulating drugs could influence $A \beta$ deposition by (a) directly influencing $A \beta$ production through alterations in secretase activity, (b) directly altering $A \beta$ deposition, or (c) indirectly influencing $A \beta$ deposition by altering levels of factors such as apoE. Alternatively, it is possible that the beneficial effect of cholesterol-lowering drugs on $\mathrm{AD}$ is related not to effects on $A \beta$, but rather to the fact that a CNS ischemic event can convert preclinical AD to clinically diagnosable dementia (64). It is worth noting that in a prospective population-based study, high systolic blood pressure was associated with a higher relative risk for AD than elevated serum cholesterol levels were (56). Nevertheless, regardless of the mechanism, treatment with statins or other cholesterol-altering agents may have a significant clinical benefit in the prevention of AD.

The complex interaction of cholesterol with $A \beta$ indicates that there are many potential ways to alter $A \beta$ metabolism. Other examples of the complex effects of drugs on $A \beta$ metabolism include the action of the PI3K inhibitor wortmannin (65). Wortmannin inhibits $A \beta$ production, both in cells and in vivo, apparently by altering APP trafficking. Although such drugs do not selectively target $A \beta$, if these compounds are relatively nontoxic (which is not the case for wortmannin), they are reasonable candidates for anti-A $\beta$ therapy.

Therapies targeting $A \beta$ aggregation or removal. Because $A \beta$ aggregation appears essential for the initiation of the $\mathrm{AD}$ pathogenic cascade, it may also be possible to prevent $A D$ by altering $A \beta$ aggregation or removing aggregates that are already formed. A number of research groups are currently exploring the development of direct $A \beta$ aggregation inhibitors (66). While some encouraging results have been reported in animal models $(67,68)$, these compounds are peptide-like and unlikely to make good drugs. An alternative strategy for altering $A \beta$ aggregation was reported recently. In APP transgenic mice treated with clioquinol (an antibiotic and bioavailable $\mathrm{Cu} / \mathrm{Zn}$ chelator), marked reduction in $\mathrm{A} \beta$ deposition occurred after several months of treatment (69). Zinc and other divalent cations appear necessary for A $\beta$ aggregation (70). Thus, metal chelation may have some therapeutic benefit in the treatment of $\mathrm{AD}$, either by preventing $\mathrm{A} \beta$ aggregation or by disrupting preformed aggregates. Clioquinol is a reasonably well tolerated drug in humans and is currently in a phase II clinical trial for AD.

One of the most surprising developments in anti-A $\beta$ therapy is $A \beta$ immunization. Direct immunization with aggregated $A \beta 42$ was originally shown to attenuate $A \beta$ deposition significantly in APP transgenic mice (71). A $\beta$ immunization now appears to be effective in reducing amyloid deposition in multiple mouse models when mice are immunized, either actively with $A \beta$, or passively with intact anti-A $\beta$ antibodies (72-76). However, it appears that there are some limits to the ability of immunization to clear existing plaques. Immunization of mice with large initial amyloid loads does not have a significant impact on amyloid deposition (76). Whether this lack of clearance can be attributed to an inherent limitation of the immunization approach or to the lack of production of sufficient amounts of anti-A $\beta$ to clear large amounts of $A \beta$ is unknown. In the latter case, one would postulate that simply increasing the amount of anti-A $\beta$ would cause more $A \beta$ to be cleared. Significantly, several groups have shown that, even in the apparent absence of any effect on $A \beta$ load in the brain, $A \beta$ immunization can ameliorate a cognitive deficit in reference memory and working spatial memory in APP transgenic mice (74, 77). This suggests that, even in the absence of $A \beta$ reduction, immunization may have some therapeutic effect. However, given that the relationship between memory deficits observed in these mice and those in humans with $\mathrm{AD}$ is unknown, the significance of this behavioral correction in mice is unclear.

Of interest are experiments showing that the local application of anti-A $\beta$ to the brain can result in rapid clearance and resolution of the plaques, along with a robust microglial infiltration and activation (78). Based on these observations, it may be possible to rapidly clear existing $A \beta$ deposits, at least in mouse models, given sufficient local concentrations of anti-A $\beta$ in the brain. While questions regarding mechanisms abound, it is thought that antibodies to $A \beta$ do one or more of 
the following: (a) enhance clearance of $A \beta$, (b) disrupt $A \beta$ fibrils, (c) prevent $A \beta$ fibril formation, and/or (d) block the toxic effects of $A \beta$ aggregates.

Although the initial phase I trial of $A \beta 42$ immunization in humans was well tolerated, the discontinuation of the phase II trial due to meningio-encephalitic presentation in about $5 \%$ of the study group represents a severe setback for direct immunization strategies. Unfortunately, due to the paucity of information on the nature of the side effects, all hypotheses regarding the nature of the postvaccination syndrome remain highly speculative (79). Very recent data now show that one patient with the postvaccination syndrome did have modest anti-A $\beta$ titers and high levels of anti- $\mathrm{A} \beta$ antibodies in the cerebrospinal fluid (CSF) $(80,81)$. This patient did respond to steroid-induced immunosuppression, but the anti-A $\beta$ titers remained unchanged after recovery. Although it has been suggested that the high CSF anti-A $\beta$ titers caused disease in this individual, it is equally likely that the high CSF titers were the result of the meningio-encephalitic presentation, perhaps due to a $T$ cell response against $A \beta$ or APP.

Studies of $A \beta$ metabolism reveal a number of potential therapeutic strategies that may alter $A \beta$ accumulation in the $\mathrm{AD}$ brain. Agents targeting $\mathrm{A} \beta$-induced cascades are also being evaluated; however, it is much more difficult to determine the potential efficacy of these, since the APP mouse models do not demonstrate all of the pathological features apparent in the AD brain. Moreover, because of the lack of clarity regarding how $A \beta$ leads to neuronal dysfunction and death, most therapeutic modalities targeting downstream effects of $A \beta$ are not necessarily specific to $\mathrm{AD}$. Thus, agents such as antioxidants, neurotrophic factors, apoptosis inhibitors, and other neuroprotective agents may all be of benefit in the treatment of $\mathrm{AD}$. They are also likely to be of general utility in other neurodegenerative conditions.

One intriguing modality currently being considered for AD treatment is the use of NSAIDs. Multiple epidemiologic studies support a role for the use of NSAIDs in preventing the development of AD. Based on the known mechanism of action of NSAIDs and the evidence for an $A \beta$-induced inflammatory cascade in the $\mathrm{AD}$ brain, it is proposed that the anti-inflammatory property of these drugs is responsible for their apparent benefit to patients with $\mathrm{AD}$. The recent data demonstrating that some NSAIDs can selectively lower $\mathrm{A} \beta 42$ raise the possibility that this mechanism, rather than the anti-inflammatory property of these compounds, confers protection (49). Alternatively, it may be the anti-inflammatory property, or a combination of the anti-inflammatory and $A \beta 42$-lowering properties, that confers protection. Significantly, several NSAIDs are currently being tested for efficacy in either treating or preventing AD. However, only one of the current trials is using an NSAID, ibuprofen, that potentially lowers $A \beta 42$.

\section{Primary prevention or therapeutic intervention?}

The epidemiologic data supporting a protective role for both statins and NSAIDs in AD indicate that primary prevention of $\mathrm{AD}$ may be feasible. Whether therapeutic intervention is likely to have a disease-modifying effect is much more controversial. Definitive insight into the temporal progression of $\mathrm{AD}$ is lacking. However, evidence (gathered from the study of AD-like pathological features in the brains of patients with Down syndrome) suggests that $A \beta$ accumulation precedes clinical cognitive impairment by many years, or even decades (82). Moreover, recent data using registered volumetric MRI show that brain atrophy begins prior to the onset of symptoms in carriers of AD-linked PS mutations $(83,84)$. If $A \beta$ is deposited for years before symptoms appear, then what is the trigger for the cognitive decline? Since the trigger is unknown, it is quite likely that anti-A $\beta$ therapy will be more effective when implemented prior to the onset of disease symptoms.

\section{Predicting the risk for development of AD}

Although it is possible that anti-A $\beta$ agents will demonstrate disease modification when they are given to patients with early stages of $\mathrm{AD}$ or mild cognitive impairment, failure to show efficacy in these patients should not prevent further study of these drugs' usefulness in the prevention of AD. However, several obstacles will impede the rapid testing of agents in preventive trials if they fail in initial therapeutic trials. The first is that any drug used for primary prevention must be safe. Because of this, drugs such as statins and NSAIDs, which are well tolerated, will likely be tested in a preventative paradigm before novel secretase inhibitors or immunization approaches are tested. The second obstacle centers on the lack of ability to predict who is likely to develop AD. Thousands of subjects are needed for primary prevention studies, which are therefore both difficult to conduct and very expensive. Improvement in the ability to predict who is likely to develop the disease will allow trials on only those individuals at high risk for $\mathrm{AD}$, clearly helping primary prevention studies. Although predictive tests for $\mathrm{AD}$ are lagging behind therapeutic advances, future developments in $\mathrm{AD}$ genetics, discovery or validation of predictive biomarkers, and advances in imaging are likely to identify individuals at high risk for development of $\mathrm{AD}$, thus enabling primary prevention trials to be conducted in a more cost-effective and efficient manner. Identifying genes that confer risk for $L O A D$. Concordance rates in LOAD between identical twins are quite high, suggesting that $\mathrm{AD}$ has a very significant genetic component $(85,86)$. Moreover, family history of $\mathrm{AD}$ is a very important risk factor. ApoE4, located on chromosome 19 , is currently the only established genetic risk factor for LOAD. However, more than $50 \%$ of individuals who develop LOAD lack an apoE4 allele, and some apoE4 homozygotes do not develop AD (87). Additional potential AD-risk genes have been identified on chromosomes 6, 9, 10, and 12; association studies also implicate other candidate genes as risk factors for $\mathrm{AD}(88)$. A locus on chromosome 10 is currently under intense scrutiny, because three independent analyses identify this region as one likely to contain a gene or genes that contributes significantly to AD risk (89-91). Of note, 
one of these studies found evidence for linkage using plasma $A \beta 42$ levels as an intermediate phenotype for $\mathrm{AD}(90)$. New data on the human genome will speed discovery of genes involved in LOAD. In the future, analyses of multiple genes will play a significant role in identifying individuals at high risk for developing AD.

Predictive biomarkers. CSF A $\beta$, tau, and isoprostane measurements are receiving attention as potential diagnostic biomarkers for AD (92-95). Given the accuracy of current clinical diagnoses of $\mathrm{AD}$, such diagnostic procedures are not likely to significantly impact the management of $\mathrm{AD}$. What is really needed is a predictive biomarker that can be used to predict risk for developing $\mathrm{AD}$, and also to monitor treatment. Although measurement of plasma $A \beta$ levels is still a long way from finding a place in the clinical management of $\mathrm{AD}$, there is emerging evidence that such measurements may be useful, both in predicting risk for developing $\mathrm{AD}$ and for monitoring anti-A $\beta$ therapy $(90,96)$. Plasma $\mathrm{A} \beta 42$ levels are elevated in patients with AD-causing mutations in APP, PS1, or PS2 (12). In at least one study, patients with higher plasma $A \beta$ levels developed $\mathrm{AD}$ more rapidly than those with lower $\mathrm{A} \beta$ levels (97, 98). Moreover, it appears that plasma $A \beta$ levels are at least partially genetically determined (96). Ongoing prospective studies should enable a more complete evaluation of the potential role of plasma $A \beta$ as a predictive biomarker for the development of AD.

Imaging. If $\mathrm{A} \beta$ deposition precedes clinical $\mathrm{AD}$ by many years, it should be possible to monitor presymptomatic amyloid deposition. Recent studies show that $\mathrm{A} \beta$ can be imaged in transgenic mice, following peripheral administration of amyloid-binding compounds, which suggests that imaging of amyloid deposits in living patients will be feasible in the near future $(78,99$, 100). Moreover, advances in MRI resolution may one day make imaging amyloid as routine as MRI scans are today $(101,102)$. Such advances will be extremely useful, because they may identify individuals at risk for imminent development of AD.

\section{Monitoring disease progression using biomarkers and imaging}

Any disease-modifying therapy must show clinical efficacy in order to be approved for the treatment or prevention of $\mathrm{AD}$. This means that the rate of cognitive decline must be decreased or halted. Until that hurdle is overcome, ancillary studies examining effects of any therapy on $A \beta$ or other biomarker levels in the plasma or CSF, on amyloid load in the brain, or on brain atrophy are largely meaningless. Once a convincing link is established between changes in any of these biomarkers and a clinically appreciable disease-modifying effect, approval of future drugs, which work through a similar mechanism, may require only that they modify linked biomarkers. For this reason, it is extremely important to monitor these parameters in current and future clinical trials. Studies with novel agents that assess both biomarkers and cognitive outcomes may be much more informative than studies that only assess clinical outcomes.

\section{Summary}

Cognitive enhancers that target acetylcholinesterase remain the only FDA-approved therapies for the treatment of the cognitive decline in AD. Such therapy is unlikely to modify the course of the disease to any significant extent. In contrast, therapies currently being developed that are based on an increased understanding of the pathogeneses of $\mathrm{AD}$ are likely to have disease-modifying effects. Given the plethora of potential targets, it is likely that successful anti-A $\beta$ therapies will emerge. The major challenge that remains is to show that such therapies actually alter cognitive decline in humans. The medical community should be cautious in evaluating the efficacy of anti-A $\beta$ drugs, as they may not show such disease-modifying effects when given in therapeutic trials. To restate the analogy to atherosclerotic disease, by the time a patient is experiencing angina, the patient needs a bypass or angioplasty, not a cholesterol-lowering agent (although after intervention such an agent would be appropriate). Similarly, in $\mathrm{AD}$, by the time a patient is symptomatic, $A \beta$-lowering therapies may not be effective. We must hope that advances in diagnostic prediction and monitoring of disease progression proceed with a pace that equals the advances currently being made in developing $\mathrm{AD}$ therapeutics that target $\mathrm{A} \beta$. If they do, then it is likely that $\mathrm{AD}$ will become manageable through a combination of presymptomatic screening, early therapeutic intervention, and vigilant monitoring of the effectiveness of treatment.

1. Small, G.W., et al. 1997. Diagnosis and treatment of Alzheimer disease and related disorders. Consensus statement of the American Association for Geriatric Psychiatry, the Alzheimer's Association, and the American Geriatrics Society. JAMA. 278:1363-1371.

2. Rice, D.P., et al. 2001. Prevalence, costs, and treatment of Alzheimer's disease and related dementia: a managed care perspective. Am. J. Manag. Care. 7:809-818.

3. Fillit, H., Hill, J.W., and Fufferman, R. 2002. Health care utilization and costs of Alzheimer's disease: the role of co-morbid conditions, disease stage, and pharmacotherapy. Fam. Med. 34:528-535.

4. Crook, R., et al. 1998. A variant of Alzheimer's disease with spastic paraparesis and unusual plaques due to deletion of exon 9 of presenilin 1. Nat. Med. 4:452-455.

5. Kwok, J.B., et al. 1997. Two novel (M233T and R278T) presenilin-1 mutations in early-onset Alzheimer's disease pedigrees and preliminary evidence for association of presenilin-1 mutations with a novel phenotype. Neuroreport. 8:1537-1542.

6. Golde, T.E., Eckman, C.B., and Younkin, S.G. 2000. Biochemical detection of $A \beta$ isoforms: implications for pathogenesis, diagnosis, and treatment of Alzheimer's disease. Biochim. Biophys. Acta. 1502:172-187.

7. Golde, T.E., and Younkin, S.G. 2001. Presenilins as therapeutic targets for the treatment of Alzheimer's disease. Trends Mol. Med. 7:264-269.

8. Hardy, J. 1997. Amyloid, the presenilins and Alzheimer's disease. Trends Neurosci. 20:154-159.

9. Younkin, S.G. 1998. The role of A beta 42 in Alzheimer's disease. J. Physiol. Paris. 92:289-292.

10. Selkoe, D.J. 2001. Alzheimer's disease: genes, proteins, and therapy. Physiol. Rev. 81:741-766.

11. Nilsberth, C., et al. 2001. The 'Arctic' APP mutation (E693G) causes Alzheimer's disease by enhanced Abeta protofibril formation. Nat. Neurosci. 4:887-893.

12. Scheuner, D., et al. 1996. Secreted amyloid beta-protein similar to that in the senile plaques of Alzheimer's disease is increased in vivo by the presenilin 1 and 2 and APP mutations linked to familial Alzheimer's disease. Nat. Med. 2:864-870.

13. Holtzman, D.M., et al. 2000. Apolipoprotein E facilitates neuritic and cerebrovascular plaque formation in an Alzheimer's disease model. Ann. Neurol. 47:739-747.

14. Jarrett, J.T., Berger, E.P., and Lansbury, P.T., Jr. 1993. The carboxy terminus of $\beta$ amyloid protein is critical for the seeding of amyloid formation: impli- 
cations for pathogenesis of Alzheimer's disease. Biochemistry. 32:4693-4697. 15. Price, D.L., and Sisodia, S.S. 1998. Mutant genes in familial Alzheimer's disease and transgenic models. Annu. Rev. Neurosci. 21:479-505.

16. Yankner, B.A. 1996. Mechanisms of neuronal degeneration in Alzheimer's disease. Neuron. 16:921-932.

17. Vidal, R., et al. 1999. A stop-codon mutation in the BRI gene associated with familial British dementia. Nature. 399:776-781.

18. Vidal, R., et al. 2000. A decamer duplication in the $3^{\prime}$ region of the BRI gene originates an amyloid peptide that is associated with dementia in a Danish kindred. Proc. Natl. Acad. Sci. USA. 97:4920-4925.

19. Hardy, J.A., and Higgins, G.A. 1992. Alzheimer's disease: the amyloid cascade hypothesis. Science. 256:184-185.

20. Lambert, M.P., et al. 1998. Diffusible, nonfibrillar ligands derived from Abeta1-42 are potent central nervous system neurotoxins. Proc. Natl. Acad. Sci. USA. 95:6448-6453.

21. Walsh, D.M., Lomakin, A., Benedek, G.B., Condron, M.M., and Teplow, D.B. 1997. Amyloid beta-protein fibrillogenesis. Detection of a protofibrillar intermediate. J. Biol. Chem. 272:22364-22372.

22. Lashuel, H.A., Hartley, D., Petre, B.M., Walz, T., and Lansbury, P.T., Jr. 2002. Neurodegenerative disease: amyloid pores from pathogenic mutations. Nature. 418:291.

23. LaFerla, F.M., Tinkle, B.T., Bieberich, C.J., Haudenschild, C.C., and Jay, G. 1995. The Alzheimer's A beta peptide induces neurodegeneration and apoptotic cell death in transgenic mice. Nat. Genet. 9:21-30.

24. Gouras, G.K., et al. 2000. Intraneuronal Abeta42 accumulation in human brain. Am. J. Pathol. 156:15-20.

25. Price, D.L., Tanzi, R.E., Borchelt, D.R., and Sisodia, S.S. 1998. Alzheimer's disease: genetic studies and transgenic models. Annu. Rev. Genet. 32:461-493.

26. Small, D.H., Mok, S.S., and Bornstein, J.C. 2001. Alzheimer's disease and Abeta toxicity: from top to bottom. Nat. Rev. Neurosci. 2:595-598.

27. Akiyama, H., et al. 2000. Inflammation and Alzheimer's disease. Neurobiol. Aging. 21:383-421.

28. Varadarajan, S., Yatin, S., Aksenova, M., and Butterfield, D.A. 2000 Review: Alzheimer's amyloid beta-peptide-associated free radical oxidative stress and neurotoxicity. J. Struct. Biol. 130:184-208.

29. Lee, V.M.-Y., and Trojanowski, J.Q. 1992. The disordered neuronal cytoskeleton in Alzheimer's disease. Curr. Opin. Neurobiol. 2:653-656.

30. Hutton, M., et al. 1998. Association of missense and $5^{\prime}$-splice-site mutations in tau with the inherited dementia FTDP-17. Nature. 393:702-705.

31. Spillantini, M.G., et al. 1998. Mutation in the tau gene in familial multiple system tauopathy with presenile dementia. Proc. Natl. Acad. Sci. USA 95:7737-7741.

32. Lewis, J., et al. 2000. Neurofibrillary tangles, amyotrophy and progressive motor disturbance in mice expressing mutant $(\mathrm{P} 301 \mathrm{~L})$ tau protein. Nat. Genet. 25:402-405

33. Gotz, J., Chen, F., van Dorpe, J., and Nitsch, R.M. 2001. Formation of neurofibrillary tangles in P301l tau transgenic mice induced by Abeta 42 fibrils. Science. 293:1491-1495.

34. Lewis, J., et al. 2001. Enhanced neurofibrillary degeneration in transgenic mice expressing mutant tau and APP. Science. 293:1487-1491.

35. Evin, G., et al. 2001. Aspartyl protease inhibitor pepstatin binds to the presenilins of Alzheimer's disease. Biochemistry. 40:8359-8368.

36. Li, Y.M., et al. 2000. Photoactivated gamma-secretase inhibitors directed to the active site covalently label presenilin 1. Nature. 405:689-694.

37. Esler, W.P., et al. 2000. Transition-state analogue inhibitors of gammasecretase bind directly to presenilin-1. Nat. Cell Biol. 2:428-434.

38. Seiffert, D., et al. 2000. Presenilin-1 and -2 are molecular targets for gamma-secretase inhibitors. J. Biol. Chem. 275:34086-34091.

39. Dovey, H., Varghese, J., and Anderson, J.P. 2000. Functional gamma-secretase inhibitors reduce beta-amyloid peptide levels in the brain. J. Neurochem. 76:1-10.

40. De Strooper, B., et al. 1999. A presenilin-1-dependent gamma-secretaselike protease mediates release of Notch intracellular domain. Nature. 398:518-522.

41. Hadland, B.K., et al. 2001. Gamma-secretase inhibitors repress thymocyte development. Proc. Natl. Acad. Sci. USA. 98:7487-7491.

42. Ni, C.Y., Murphy, M.P., Golde, T.E., and Carpenter, G. 2001. $\gamma$-Secretase cleavage and nuclear localization of ErbB-4 receptor tyrosine kinase. Science. 294:2179-2181.

43. Kammesheidt, A., et al. 1992. Deposition of $\beta / A 4$ immunoreactivity and neuronal pathology in transgenic mice expressing the carboxyl-terminal fragment of the Alzheimer amyloid precursor in the brain. Proc. Natl. Acad. Sci. USA. 89:10857-10861.

44. Yankner, B.A., et al. 1989. Neurotoxicity of a fragment of the amyloid precursor associated with Alzheimer's disease. Science. 245:417-420.

45. Cai, H., et al. 2001. BACE1 is the major beta-secretase for generation of Abeta peptides by neurons. Nat. Neurosci. 4:233-234.

46. Luo, Y., et al. 2001. Mice deficient in BACE1, the Alzheimer's beta-secretase, have normal phenotype and abolished beta-amyloid generation. Nat. Neurosci. 4:231-232.
47. Hong, L., et al. 2002. Crystal structure of memapsin 2 (beta-secretase) in complex with an inhibitor OM00-3. Biochemistry. 41:10963-10967.

48. Hong, L., et al. 2000. Structure of the protease domain of memapsin 2 (beta-secretase) complexed with inhibitor. Science. 290:150-153.

49. Weggen, S., et al. 2001. A subset of NSAIDs lower amyloidogenic Abeta42 independently of cyclooxygenase activity. Nature. 414:212-216.

50. Lim, G.P., et al. 2000. Ibuprofen suppresses plaque pathology and inflammation in a mouse model for Alzheimer's disease. J. Neurosci. 20:5709-5714.

51. Wolozin, B., Kellman, W., Ruosseau, P., Celesia, G.G., and Siegel, G. 2000 Decreased prevalence of Alzheimer disease associated with 3-hydroxy-3methyglutaryl coenzyme A reductase inhibitors. Arch. Neurol. 57:1439-1443.

52. Jick, H., Zornberg, G.L., Jick, S.S., Seshadri, S., and Drachman, D.A. 2000 Statins and the risk of dementia. Lancet. 356:1627-1631.

53. Jarvik, G.P., et al. 1995. Interactions of apolipoprotein E genotype, total cholesterol level, age, and sex in prediction of Alzheimer's disease: a casecontrol study. Neurology. 45:1092-1096.

54. Notkola, I.L., et al. 1998. Serum total cholesterol, apolipoprotein E epsilon 4 allele, and Alzheimer's disease. Neuroepidemiology. 17:14-20.

55. Hofman, A., et al. 1997. Atherosclerosis, apolipoprotein E, and prevalence of dementia and Alzheimer's disease in the Rotterdam Study. Lancet. 349:151-154.

56. Kivipelto, M., et al. 2001. Midlife vascular risk factors and Alzheimer's disease in later life: longitudinal, population based study. BMJ. 322:1447-1451

57. Simons, M., et al. 1998. Cholesterol depletion inhibits the generation of beta-amyloid in hippocampal neurons. Proc. Natl. Acad. Sci. USA. 95:6460-6464.

58. Refolo, L.M., et al. 2001. A cholesterol-lowering drug reduces beta-amyloid pathology in a transgenic mouse model of Alzheimer's disease. Neurobiol. Dis. 8:890-899.

59. Fassbender, K., et al. 2001. Simvastatin strongly reduces levels of Alzheimer's disease beta-amyloid peptides Abeta 42 and Abeta 40 in vitro and in vivo. Proc. Natl. Acad. Sci. USA. 98:5856-5861.

60. Refolo, L.M., et al. 2000. Hypercholesterolemia accelerates the Alzheimer's amyloid pathology in a transgenic mouse model. Neurobiol. Dis. 7:321-331.

61. Puglielli, L., et al. 2001. Acyl-coenzyme A: cholesterol acyltransferase modulates the generation of the amyloid beta-peptide. Nat. Cell Biol. 3:905-912.

62. Golde, T.E., and Eckman, C.B. 2001. Cholesterol modulation as an emerging strategy for the treatment of Alzheimer's disease. Drug Discov. Today. 6:1049-1055

63. Wolozin, B. 2001. A fluid connection: cholesterol and Abeta. Proc. Natl. Acad. Sci. USA. 98:5371-5373.

64. Snowdon, D.A., et al. 1997. Brain infarction and the clinical expression of Alzheimer disease. The Nun Study. JAMA. 277:813-817.

65. Haugabook, S.J., et al. 2001. Reduction of Abeta accumulation in the Tg2576 animal model of Alzheimer's disease after oral administration of the phosphatidyl-inositol kinase inhibitor wortmannin. FASEB J. 15:16-18

66. Soto, C. 1999. Plaque busters: strategies to inhibit amyloid formation in Alzheimer's disease. Mol. Med. Today. 5:343-350.

67. Permanne, B., et al. 2002. Reduction of amyloid load and cerebral damage in a transgenic mouse model of Alzheimer's disease by treatment with a beta-sheet breaker peptide. FASEB J. 16:860-862.

68. Sigurdsson, E.M., Permanne, B., Soto, C., Wisniewski, T., and Frangione, B. 2000. In vivo reversal of amyloid-beta lesions in rat brain. J. Neuropathol. Exp. Neurol. 59:11-17.

69. Cherny, R.A., et al. 2001. Treatment with a copper-zinc chelator markedly and rapidly inhibits beta-amyloid accumulation in Alzheimer's disease transgenic mice. Neuron. 30:665-676.

70. Curtain, C.C., et al. 2001. Alzheimer's disease amyloid-beta binds copper and zinc to generate an allosterically ordered membrane-penetrating structure containing superoxide dismutase-like subunits. J. Biol. Chem. 276:20466-20473.

71. Schenk, D., et al. 1999. Immunization with amyloid-beta attenuates Alzheimer-disease-like pathology in the PDAPP mouse. Nature. 400:173-177.

72. Lemere, C.A., et al. 2000. Nasal A beta treatment induces anti-A beta antibody production and decreases cerebral amyloid burden in PD-APP mice. Ann. NY Acad. Sci. 920:328-331.

73. DeMattos, R.B., et al. 2001. Peripheral anti-A beta antibody alters CNS and plasma A beta clearance and decreases brain A beta burden in a mouse model of Alzheimer's disease. Proc. Natl. Acad. Sci. USA. 98:8850-8855.

74. Janus, C., et al. 2000. A beta peptide immunization reduces behavioural impairment and plaques in a model of Alzheimer's disease. Nature. 408:979-982.

75. Bard, F., et al. 2000. Peripherally administered antibodies against amy- 
loid beta-peptide enter the central nervous system and reduce pathology in a mouse model of Alzheimer disease. Nat. Med. 6:916-919.

76. Das, P., Murphy, M.P., Younkin, L.H., Younkin, S.G., and Golde, T.E. 2001. Reduced effectiveness of Abeta1-42 immunization in APP transgenic mice with significant amyloid deposition. Neurobiol. Aging. 22:721-727.

77. Morgan, D., et al. 2000. A beta peptide vaccination prevents memory loss in an animal model of Alzheimer's disease. Nature. 408:982-985.

78. Bacskai, B.J., et al. 2001. Imaging of amyloid-beta deposits in brains of living mice permits direct observation of clearance of plaques with immunotherapy. Nat. Med. 7:369-372.

79. Das, P., and Golde, T.E. 2002. Open peer commentary regarding Abeta immunization and CNS inflammation by Pasinetti et al. Neurobiol. Aging. 23:671-674.

80. Hock, C., et al. 2002. Generation of antibodies specific for beta-amyloid by vaccination of patients with Alzheimer disease. Nat. Med. 8:1270-1275.

81. Schenk, D. 2002. Opinion. Amyloid-beta immunotherapy for Alzheimer's disease: the end of the beginning. Nat. Rev. Neurosci. 3:824-828.

82. Lemere, C.A., et al. 1996. Sequence of deposition of heterogeneous amyloid beta-peptides and APO E in Down syndrome: implications for initial events in amyloid plaque formation. Neurobiol. Dis. 3:16-32.

83. Chan, D., et al. 2001. Rates of global and regional cerebral atrophy in $\mathrm{AD}$ and frontotemporal dementia. Neurology. 57:1756-1763.

84. Fox, N.C., et al. 2001. Imaging of onset and progression of Alzheimer's disease with voxel-compression mapping of serial magnetic resonance images. Lancet. 358:201-205.

85. Breitner, J.C., et al. 1994. Alzheimer's disease in the NAS-NRC registry of aging twin veterans. II. Longitudinal findings in a pilot series. National Academy of Sciences. National Research Council Registry. Dementia. 5:99-105.

86. Rubinsztein, D.C. 1997. The genetics of Alzheimer's disease. Prog. Neurobiol. 52:447-454.

87. Roses, A.D. 1997. Apolipoprotein E, a gene with complex biologica interactions in the aging brain. Neurobiol. Dis. 4:170-185.

88. Kehoe, P., et al. 1999. A full genome scan for late onset Alzheimer's disease. Hum. Mol. Genet. 8:237-245.
89. Myers, A., et al. 2000. Susceptibility locus for Alzheimer's disease on chromosome 10. Science. 290:2304-2305.

90. Ertekin-Taner, N., et al. 2000. Linkage of plasma Abeta42 to a quantitative locus on chromosome 10 in late-onset Alzheimer's disease pedigrees. Science. 290:2303-2304.

91. Bertram, L., et al. 2000. Evidence for genetic linkage of Alzheimer's disease to chromosome 10q. Science. 290:2302-2303.

92. Galasko, D. 2001. Biological markers and the treatment of Alzheimer's disease. J. Mol. Neurosci. 17:119-125.

93. Boss, M.A. 2000. Diagnostic approaches to Alzheimer's disease. Biochim. Biophys. Acta. 1502:188-200.

94. Pratico, D., et al. 2000. Increased 8,12-iso-iPF2alpha-VI in Alzheimer's disease: correlation of a noninvasive index of lipid peroxidation with disease severity. Ann. Neurol. 48:809-812.

95. Montine, T.J., et al. 2001. Cerebrospinal fluid abeta42, tau, and f2-isoprostane concentrations in patients with Alzheimer disease, other dementias, and in age-matched controls. Arch. Pathol. Lab. Med. 125:510-512

96. Ertekin-Taner, N., et al. 2001. Heritability of plasma amyloid beta in typical late-onset Alzheimer's disease pedigrees. Genet. Epidemiol. 21:19-30.

97. Mayeux, R., et al. 1999. Plasma amyloid beta-peptide 1-42 and incipient Alzheimer's disease. Ann. Neurol. 46:412-416.

98. Schupf, N., et al. 2001. Elevated plasma amyloid beta-peptide 1-42 and onset of dementia in adults with Down syndrome. Neurosci. Lett. 301:199-203.

99. Skovronsky, D.M., et al. 2000. In vivo detection of amyloid plaques in a mouse model of Alzheimer's disease. Proc. Natl. Acad. Sci. USA. 97:7609-7614.

100.Klunk, W.E., et al. 2002. Imaging Abeta plaques in living transgenic mice with multiphoton microscopy and methoxy-X04, a systemically administered Congo red derivative. J. Neuropathol. Exp. Neurol. 61:797-805.

101.Jack, C.R., Jr., et al. 2002. Antemortem MRI findings correlate with hippocampal neuropathology in typical aging and dementia. Neurology. 58:750-757.

102.Bacskai, B.J., Klunk, W.E., Mathis, C.A., and Hyman, B.T. 2002. Imaging amyloid-beta deposits in vivo. J. Cereb. Blood Flow Metab. 22:1035-1041. 